\title{
Cognitive processing and time perception
}

\author{
EWART A. C. THOMAS and WANDA B. WEAVER \\ Stanford University, Stanford, California 94305
}

\begin{abstract}
When a visual field is presented for 40 or $80 \mathrm{msec}$ and a subject is asked to judge the duration of the stimulus, judged duration is found to be less when the field is blank than when the field contains three letters, but is the same whether the three letters form a word or not. The perceived difference between "filled" and "blank" fields increases when the subject is required to memorize the presented letters. These data are consistent with a theory which assumes, inter alia, that a stimulus is analyzed by a visual information processor and a timer, that attention is shared between these processors, and that temporal judgments are based on the output of both processors.
\end{abstract}

Most, if not all, theories about how temporal judgments are made contain the assumption that the perceived length of an interval depends on the "information" occurring during that interval. Because of this assumption, these theories depend on theories about how stimulus information is processed, stored, and retrieved within an organism. In the simplest case, where the stimulus consists, for example. of two 10-msec clicks separated by an interval, $t$, and the subject's task is to estimate $t$, the assumption has been made that the relevant stimulus information is the number of subjective pulses occurring during t. Various properties of the judgments of these "empty" intervals can be derived after assumptions are made about the pulse rate and the fluctuation of this rate (Creelman. 1962; Kinchla, 1972; Treisman, $1963)$.

When the temporal interval is "filled" with experimenter-given information, the task of specifying the relevant stimulus information becomes more difficult. In the case of discrete filler material, the assumption is usually retained that the number of discrete events is directly related to perceived length (Buffardi. 1971; Frankenhaeuser, 1959; Ornstein, 1969; Thomas \& Brown, 1974). In the case of information qua entropy, Michon (1965) found that perceived duration increased as response uncertainty increased but was independent of stimulus uncertainty. This led him to conclude that it was the information actively coped with in making a response, rather than the information presented for processing, which inlluenced time judgments. Finally, Ornstein (1969) proposed the theory that perceived duration is directly related to the storage size of the interval, and storage size is, in turn, directly related to the number and the complexity of events which occur in the interval. A weakness of this "storage size" theory is that there is no independent theory for determining the storage size when the stimulus information is

This work was supported partly by Grant GB-43275 from the National Science Foundation to the first author. We wish to thank A. B. Kristofferson and two referees for their helpful comments. relatively complex, e.g., linguistic.

Comparatively few studies of time perception have used durations of under $1 \mathrm{sec}$, probably because of the difficulty of tinding an adequate response measure at very short durations. Very recently, Avant, Lyman, and Antes (1975) have studied relative judgments in a paired comparison task in which visual arrays were presented for $30 \mathrm{msec}$ at varying levels of figure-ground contrast. These authors found that, as contrast increased, perceived duration increased for letter-arrays but not for random dot patterns, and they suggested that cognitive parameters, viz, stimulus familiarity, of the array can affect perceived duration. Familiarity was assumed to be inversely related to initial processing time of stimulus information, and initial processing time was assumed to be directly related to perceived duration. To account for their result that the array "I" was judged as shorter than "HIT." which was judged as shorter than "EIO," the assumption was made that "I" and "EIO" were the most and least familiar, respectively. of the three arrays.

In the present paper, we will adopt the same approach as Avant et al. (1975) to explain temporal judgments, which approach is to examine what the subject does with the presented stimulus information and then to relate perceived duration to the time spent processing some aspects of the information and/or to the "amount" of processing done. The objective of this approach is to see if processing variables, in addition to storage and retrieval variables, influence time perception. What we first need is a statement of what stimulus information is processed, how this is done, and what experimental manipulations might be expected to influence this processing.

\section{Rationale for the Present Study}

A theoretical framework for discussing time estimation has been presented by Thomas and Brown (1974), and here we will extend some of their ideas. A stimulus, consisting of information, I. presented for a 
duration. $\mathrm{t}$, is analyzed or processed at various levels. The outcome of processing is an encoding. $\mathrm{g}(\mathrm{l} ; \mathrm{t})$, of $\mathrm{I}$ and an encoding. $f(t ; \mathrm{I})$, of $t$. where $\mathrm{f}(\mathrm{t} ; \mathrm{I})$ is monotonic in $t$. For the typical task in which the subject knows the nature of the task, we assume that attention is shared among parallel processors such that, when I is "large," the timer or $f$ processor registers estimates of duration that are lower and probably more variable than when I is "small." In other words, as the timer's share of attention decreases, the average value of $f(t ; l)$ decreases and its variability probably increases. This assumption about $t(t ; l)$ is consistent with the "attention" model discussed by Thomas and Brown (1974. Example 6.1.5). We next assume that both $g(I ; t)$ and $f(t ; I)$ influence the subject's time estimate, but that, as more attention is captured by the $\mathrm{g}$ processors, the relative influence of $\mathrm{g}(\mathrm{l} ; \mathrm{t})$ increases because of the increasing unreliability of $f(t ; I)$. In the extreme case, where the subject does not know that a time estimate is required until after a "filled" duration is experienced, we assume that time estimates are derived from $g(I ; t)$ only, this because of the unreliability (even unavailability) of $f(I ; t)$. In another extreme case, where the subject knows that an estimate of an "empty" duration is required, we assume that only the encoded duration, denoted by $f(t ; 0)$. determines the time estimate. In this last case, $g(I: 1)$ may exist because of the occurrence of subjective "events," but we assume that its unreliability is large relative to that of $f(t ; 0)$ and the subject does not use it.

Given that temporal information is extracted from $g(I ; t)$, the question arises as to how this is done. As stated earlier, the storage-size theory asserts that $\mathrm{g}(\mathrm{l} ; \mathrm{t})$ consists of discrete "events" of varying complexity, and that the time estimate derived from $g$ is directly related to the number and complexity of these events. Further, Thomas and Brown (1974) suggest that the (response) time taken by the subject to give a temporal judgment might be related to the difficulty of extracting temporal information from $g$. Alternatively, theories which relate perceived duration to processing time (e.g., Avant et al., 1975) can be paraphrased as stating that the relevant aspect of $\mathrm{g}(\mathrm{I} ; \mathrm{t})$ is the encoding or memory of the time spent processing $\mathrm{I}$. Let us denote this time by $\mathrm{g}^{*}(\mathrm{I} ; \mathrm{t})$, the asterisk indicating that processing time is a particular view of the more generally conceived encoding $\mathrm{g}$. A sensitive test of this view would seem to be available when the duration to be judged $(t)$ is less than or equal to $g^{*}(I ; t)$ rather than when $t$ is much greater than $\mathrm{g}^{*}(\mathrm{I} ; \mathrm{t})$. This can be achieved either by requiring the subject to judge durations of about $100 \mathrm{msec}$, as in the study by Avant et al. (1975) or, if relatively long duratons are used, by requiring the subject to process information continuously during the interval, as in Burnside's (1971) study. In the present study, we will use very short durations.
Consider the case where a visual field is exposed for a duration t. $20 \mathrm{msec} \leqslant \mathrm{t} \leqslant 100 \mathrm{msec}$, and either is blank or contains a three-letter array. We make the important assumption that the time spent processing the letters is independent of $t$ in this range of $t$, and we denote this time by $\mathrm{g}^{*}(\mathrm{I})$ [instead of $\mathrm{g}^{*}(\mathrm{I} ; \mathrm{t})$ ]. We also assume that $g^{*}(I)$ is greater than $f^{*}(t ; I), t \leqslant 100 \mathrm{msec}$, which assumption is suggested by the numerous studies which show that visual masks interrupt processing (e.g. . Avant et al., 1975). We now have to make some assumption about how perceived duration $\tau$ depends on $\left.\mathrm{f}^{\mathrm{f}} \mathrm{t} ; \mathrm{l}\right)$ and $\mathrm{g}^{*}(\mathrm{l})$, and we make the simple one that when letters are presented, $\tau$ is a weighted average of $f$ and $\mathrm{g}^{*}$. That is.

$$
\tau(I)=a f(t: I)+(1-a) g^{*}(I), I>0, t \leqslant 100 \text { msec. (I) }
$$

where $g^{*}(I)>f(t ; I)$. When the field is blank, we assume that only $f(t ; 0)$ influences $\tau$; that is

$$
\tau(0)=f(t ; 0) .^{1}
$$

In a task where blank and filled fields are randomly interspersed in a block of trials, the share of attention given to the f processor is independent of the type of field. Therefore, for this task,

$$
f(t ; 1)=f(t ; 0) \text {. }
$$

Suppose now that the subject is required to memorize the letters for a subsequent recognition. It is reasonable to suppose that this requirement increases the share of attention given to the $\mathrm{g}$ processor and the time spent processing $I$. Because of the change in the distribution of attention, the average value and the reliability of $f(t ; I)$ should decrease, by our previous assumption. ${ }^{2}$ The effect of the decreased reliability of $f(t ; I)$ is to decrease a.

We can now consider the consequences of these assumptions when experimental data are subjected to an analysis of variance. The argument leading up to Equation 3 requires us to vary information level (I) within a block of trials. Also one would be ill-advised to keep stimulus duration $(\mathrm{T})$ constant within blocks, since the subject might realize this and respond independently of $I$ and $T$. The third factor discussed above is memory load (M), and this is varied most conveniently between blocks.

By the assumed monotonicity of $f(t ; I)$, Equations 1 and 2 imply that there should be a $\mathrm{T}$ main effect. Since $g^{*}(\mathrm{I})>f(t ; \mathrm{l})$, Equations $1-3$ imply that

$$
\tau(1)-\tau(0)=(1-a)[g *(I)-f(t ; 0)]>0,
$$

that is, that there should be an I main effect, i.e., a "filled-duration" illusion. Further, since $\mathrm{g}^{*}(\mathrm{I})$ is independent of $t$, as $f(t ; 0)$ increases towards $g^{*}(I)$ owing to an increase in $t$, the size of the illusion should 
decrease. This implies that there should be a $T$ by $I$ interaction effect. Finally, the assumed effects of introducing a memory load are that $(1-a), g^{*}(I)$, and $-f(t: 0)$ all increase, which, from Equation 4, implies that the size of the illusion should increase. Therefore, there should be an $M$ by I interaction effect. The following experiment was done to check these expectations.

\section{METHOD}

\section{Subjects}

The subjects were 20 paid undergraduates at Stanford Lntiersits.

\section{Stimuli}

The isual displays were presented on a modified three-field konix tachistoscope. Each trial was started by a 500 -msec blank ficld: this was followed by a 1 -sec dark interval, at the end of which a 40- or 80-msec tield was presented. The tield either was blank or contained three letters, which formed a word or a permutation (Perm) of a word. The two durations, 40 and $80 \mathrm{msec}$, and the three information levels. Blank. Perm, and Word, yielded six stimulus twpes. each type being presented three times in a block of trials; and nu sutject saw the same three-letter array more than once.

\section{Responses}

Each subject was trained to discriminate between blank durations of 20 msec ("short"). 60 msec ("medium"), and 100 msec ("long"). All subjects were able to do this in less than 20 trials. On each trial. the sulject was required to categorize the second (brief) duration as "shom." "medium." or "long." It was felt that this three-point response scale provided for a more accurate measurement of perctied duration than the two-point scale, "short" $\equiv 40 \mathrm{msec}$. "long" $\equiv 80$ msec.

At the end of some trial blocks. the subject had a recognition memury tent comsisting of 3 Perms and $3 W$ ords that were presented in the bluch and 3 Pernis and 3 Words that were being seen for the tir $\rightarrow$ time. The subject was required to check those items which were prescuted in the block.

\section{Procedure}

Fach subjuct was first trained to discriminate among "short." "medium." and "long" blank tields, and then was given a block of 11 prastice trials with instructions to give a time jud gment on each trial and alsu forry te memorize all the presented letter array. This sas followed by a (practice) recognition test.

Sis trial blocks. A.F. Were used; each consisted of three replications of each of the six stimulus types. plus an initial trial which was not included in any analysis. Before each block. the subject $"$ as shown an ascending and a descending series of 20-, 60-, and 100 -msec blank fields.

lrial Bhoks $A$. B. and $C$ were given with the nemory instruction and half of the subjects received these three blocks before Blocks D.F. The other half received Blocks D.F before Blecks A.C. Within thin constraint the order of blocks was varied iner sulpiects.

\section{RESULTS}

The responses "short." "medium," and "long" were cuded 0.1 . and 2. respectively, and. for each subject and trial block. the average was found of the three responses for each stimulus type. These averages were subjected to an analysis of variance for repeated measures with three replications ( $\equiv$ blocks). The

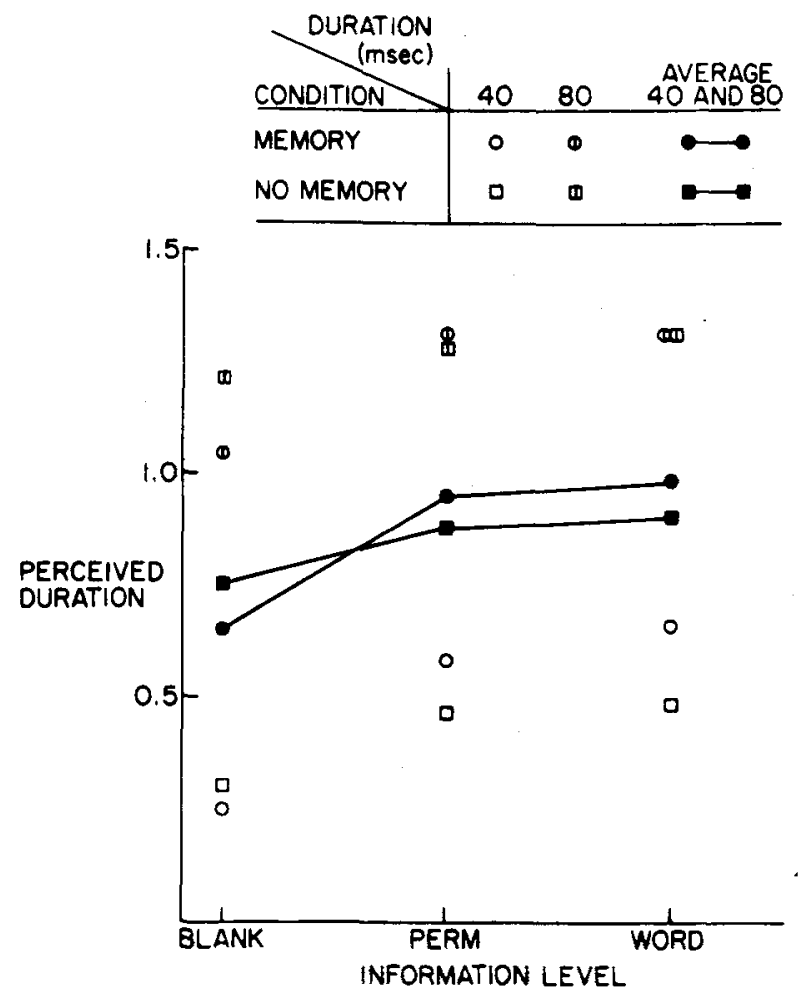

Figure 1. Temporal judgment as a function of duration, information level, and memony load.

variables of interest are duration $(\mathrm{T})$. information level (I). and memory load (M).

As expected. the $\mathrm{T}$ and $\mathrm{I}$ main effects are significant $(\mathrm{p}<.001)$. Consistent with the expectations stated at the end of the rationale. the $\mathrm{M}$ by $\mathrm{I}$ interaction is significant $[\mathrm{F}(2.38)=4.95, \mathrm{p}<.025]$. The $\mathrm{T}$ by $\mathrm{I}$ interaction is in the predicted direction. but was not signiticant $[F(1,19)=2.1 .1<p<.2]$. The data are presented graphically in Figure 1.

Since the values 0,1 , and 2 of $d$ correspond to durations of 20.60. and $100 \mathrm{msec}$. respectively. d can be converted into clock time by the formula $\tau=$ $(20+40 \mathrm{~d})$ msec. $^{3}$ The operation of the timer can best be seen by using the values of $\tau$ for blank fields in the no-memon condition, since for these stimuli $\tau$ is influenced only by the timer and in this condition maximum attention is given to the timer. For the 40-msec durations. $\tau$ is $32 \mathrm{msec}$, and for the $80-\mathrm{msec}$ durations, $\tau$ is $69 \mathrm{msec}$. Thus, there is a tendency for subjects to underestimate empty durations. but the perceived difterence between the two presented durations is very close to the actual difference.

Although performance on the recognition test has not been included in our theory. it is of some interest to see how this variable is influenced $b y T$ and $I$. The recognition dita can be analyzed in a number of nonindependent ways. Half of the items on the test were "new" and half were "old." The old items can be 
categorized as Perm or Word and as "short" (if it was presented for $40 \mathrm{msec}$ ) or "long"; new items can be categorized as Perm or Word. The responses from all subjects were pooled and the accuracy computed for judgments on each type of new item and on each type of old item. The accuracy rates for a given type of new item and the appropriate type of old item can be converted into a $\mathrm{d}^{\prime}$ measure in the conventional way.

The $d^{\prime}$ for "short" items is equal to that for "long" items, i.e., .82 (where each type of old item is compared with all new items). The d' for Words is .91 while that for Perms is .77. For Words, $\mathrm{d}^{\prime}$ is less for "short" than for "long" (.7 vs. 1.08), while for Perms the opposite is true (.9 vs. .6). Only the last of these results is surprising.

\section{DISCUSSION}

The results of this experiment, particularly the interaction between memory load and information level. are consistent with a theory which relates processing time to perceived duration. The key assumptions of this theory are that (i) in general, temporal information is obtained from both a timer ( $f$ processor) and a visual information (g) processor, (ii) attention is shared between the $f$ and $g$ processors such that the output of the $f$ processor becomes less reliable as the $\mathrm{g}$ processor captures more attention, and (iii) for the range of durations considered here, the time to process visual information is greater than and independent of duration; and perceived filled duration is a weighted average of processing time and perceived empty duration. The form of Assumption iii is thus closely tied to the design of the study and would probably be invalid if longer durations were used.

It may be noted that the Memory by Information interaction is a "cross-over" effect, with empty durations being judged as shorter when there is a memory load than when there is no load. This nonsignificant effect is consistent with our assumption that, because of the increased relative share of attention given to the $\mathrm{g}$ processor in the memory condition, the timer tends to underestimate duration. Clearly, for durations much greater than $100 \mathrm{msec}$, it may be possible for the subject to switch attention from the $g$ processor to the timer, thereby reestablishing its reliability.

Avant et al. (1975) have argued that familiarity of visual arrays is inversely related to perceived duration. Using their argument, one might have expected Perms to be judged as longer than Words, since the latter are more familiar. This difference is not found in the present study, but this may be due to the use of categorical judgments. For, as Thomas and Brown (1974) have argued, the Avant et al. result may be linked to the use of the paired comparison method-on those trials when the perceived durations of the two stimuli are the same, the judgment may be based on nontemporal variables, such as familiarity.

If the relationship were known between processing time and the outcome of processing, then the latter could be used to test a processing-time theory of time estimation. The recognition memory tests used here were part of a manipulation designed to change the distribution of attention over processors. We still entertain the hope that performance on these and similar tests would yield insights about the outcome of processing and, consequently, about the storage and retrieval of temporal information.

\section{REFERENCES}

Allan, L., Kristofferson, A. B.. \& Weins, E. W. Duration discrimination of brief light flashes. Perception \& Psychophysics, 1971. 9. 327-334.

Avant, L. L., Lyman, P. J., \& Antes, J. R. Effects of stimulus familiarity on judged visual duration. Perception \& Psychophysics, 1975, 17, 253-262.

BUfFARDI. L. Factors affecting the filled-duration illusion in the auditory, tactual, and visual modalities. Perception \& Psychophysics, 1971, 10, 292-294.

BURNSIDE, W. Judgment of short time intervals while performing mathematical tasks. Perception \& Psychophysics, 1971, 9, 404-406.

Creelman, C. D. Human discrimination of auditory duration. Journal of the Acoustical Society of America, 1962, 34, 582-593.

Frankenhaeuser. M. Estimation of time. Stockholm: Almquist \& Wiksells. 1969.

Kinchla, J. Duration discrimination of acoustically defined intervals in the 1 - to 8 -sec range. Perception \& Psychophysics. 1972, 12, 318-320.

Michon, J. A. Studies in subjective duration. II. Subjective time measurement during tasks with different information content. Acta Psychologica, 1965, 24. 205-219.

Ornstein, R. On the experience of time. Baltimore: Penguin Books, 1969.

Thomas, E. A. C., \& Brown, I. Time perception and the filled duration illusion. Perception \& Psychophysics, 1974, 16, 449.458 .

Treisman, $M$. Temporal discrimination and the indifference interval. Implications for a model of the "internal clock." Psychological Monographs. 1963, 77. No. 13.

\section{NOTES}

1. In the experiment to be reported, blank fields were illuminated tields, and Equation 2 supposes, for example, that total energy of the field does not affect perceived duration, which assumption is consistent with the data of Allan, Kristofferson, and Weins (1971)

2. Our view is that the $f$ and $g$ processors operated in parallel. An alternative view is that the f processor operates during stimulus presentation and the $\mathrm{g}$ processor starts processing at stimulus offset. According to this latter view, if a mask comes on at stimulus offset, it should prevent processing and there should be no difference in perceived duration between blank and filled fields. Avant et al. (1975) show that this difference is greater in a "mask" than in a "no-mask" condition, suggesting that this serial view of processing is unreasonable.

3. This seems to be the simplest way of relating $r$ to $d$. Alternatively, we could assume that, when a stimulus is presented, 
perceived duration is normally distributed with mean $T$ and standard deviation $\sigma$; the subject responds "short," "medium," or "long" if perceived duration is less than $60-c$, lies between $60-c$ and $60+c$, or is greater than $60+c$ msec, respectively. The frequencies of "short" and "long" responses can be converted into $z$ scores to give estimates of $(60-c-\tau) / \sigma$ and $(60+c-\tau) / \sigma . \tau$ could then be estimated if we assume some value for c (e.g., $20 \mathrm{msec})$ or for $o$.

(Received for publication September 30, 1974; revision received December 10, 1974.) 